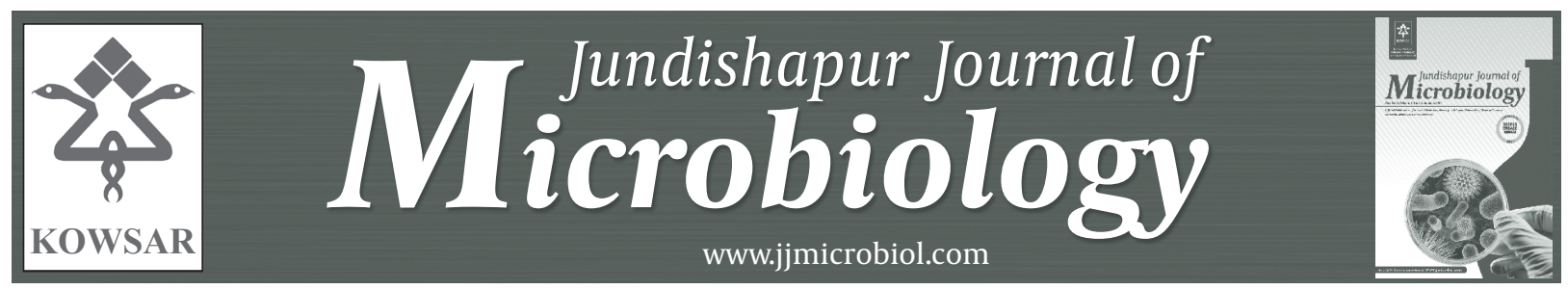

\title{
The Last Three Years Antibiotic Susceptibility Patterns of Uropathogens in Southwest of Iran
}

\author{
Ehsan Valavi ${ }^{1,{ }^{*}}$, Roya Nikfar ${ }^{1}$, Ali Ahmadzadeh ${ }^{1}$, Farshid Kompani ${ }^{1}$, Reza Najafi ${ }^{2}$, Rasool \\ Hoseini $^{2}$ \\ ${ }^{1}$ Division of Pediatric, Jundishapur University of Medical Sciences, Ahvaz, IR Iran \\ ${ }^{2}$ Faculty of Medicine, Jundishapur University of Medical Sciences, Ahvaz, IR Iran \\ *Corresponding author: Ehsan Valavi, Division of Nephrology, Pediatric Department, Abuzar Children's Hospital, Ahvaz, IR Iran.Tel: +98-9173440731, Fax: \\ +98-6114433715, E-mail:dr_ehsan_valavi@yahoo.com.
}

\begin{abstract}
A B S T R A C T
Background: Urinary tract infections are always treated empirically before the results of bacteriological cultures are obtained. The choice of antibiotics depends upon the causative organism and its expected local antibiotic susceptibility pattern.

objectives: We analyzed the spectrum and resistance patterns of uropathogens against common antimicrobial agents in Ahvaz Abuzar Children's Hospital, a tertiary care pediatric unit in southwest of Iran.

Patients and Methods: In this retrospective study, all urine samples of children hospitalized with urinary tract infection (288 patients, aged 1mon -14.5 years) during October 2008 to May 2011 were included in the study. After bacteria were identified by standard methods, antimicrobial susceptibility testing was performed using a panel of antimicrobial agents.

Results: The most of patients were girls $(\mathrm{n}=226,78.5 \%)$, and the median age was 13 months. The most common pathogens were Escherichia coli (84\%), Klebsiella spp. (10.1\%), Enterococcus spp. (2.4\%), Proteus spp. (1.7\%), and Pseudomonas spp. (1.7\%). Overall bacterial resistance spectrum was the highest for co-trimoxazole (64.8\%), followed by gentamicin (44.6\%), amikacin (40.5\%), nalidixic acid (37.3\%), cefotaxime (28.9\%), cefixime (27.5\%), ceftriaxone (27.4\%), and nitrofurantoin (10.2\%). The female:male ratio was 2:1 (67.1\% versus 32.9\%) in infants aged <1 year and 8:1 in those aged $>1$ year (89.4\% vs. 10.6\%). Vesicoureteral reflux and abnormal sonography findings were associated with high resistance to cefotaxime ( $P$ $=0.017)$, ceftriaxone $(\mathrm{P}=0.004)$, nitrofurantoin $(\mathrm{P}=0.014)$, and nalidixic acid $(\mathrm{P}<0.001)$.

Conclusions: Increasing resistance to third-generation cephalosporins changed our opinion for using them as a single empiric intravenous therapy in hospitalized and very ill patients with acute pyelonephritis; the success will be achieved by concomitant use of an aminoglycoside or using other potent antibiotics.
\end{abstract}

Keywords: Antibiotic susceptibility; Children; Urinary Tract Infections

Copyright ( (2013, Ahvaz Jundishapur University of Medical Sciences; Published by Kowsar Corp.

Article type: Research Article; Received: 25 Mar 2012; Revised: 27 May 2012; Accepted: 20 Jun 2012; Epub: 01 Jun 2013 ; Ppub: Jun 2013

Implication for health policy/practice/research/medical education:

Detection of the resistance pattern of uropathogens to antimicrobial agents is important for clinical and epidemiological point of view and this reporting in our area will help physicians to select appropriate antimicrobials for empirical therapy.

PPlease cite this paper as:

Valavi E, Nikfar R, Ahmadzadeh A, Kompani F, Najafi R, Hoseini R. The Last Three Years Antibiotic Susceptibility Patterns of Uropathogens in Southwest of Iran. Jundishapur J Microbiol. 2013;6(4):e4958. DOI: 10.5812/jjm.4958.

Copyright @ 2013, Ahvaz Jundishapur University of Medical Sciences; Published by Kowsar Corp.

This is an Open Access article distributed under the terms of the Creative Commons Attribution License (http://creativecommons.org/licenses/by/3.0), which permits unrestricted use, distribution, and reproduction in any medium, provided the original work is properly cited. 


\section{Background}

Although urinary tract infection (UTI) is one of the most common bacterial diseases in children, its diagnosis is often delayed due to obscure clinical findings. In pediatric patients, acute pyelonephritis may be associated with high morbidity and long-term complications like renal scarring, hypertension, and chronic renal failure (1-3). Gram-negative enteric bacilli, especially Escherichia coli and Klebsiella spp., are the leading pathogens, though Enterococcus spp., yeasts, and Staphylococcus spp. have recently emerged as prominent agents.

\section{Objectives}

Resistance to aminoglycosides, cephalosporins, and fluoroquinolones - the most preferred agents for empiric therapy of UTIs-has increased markedly in these recent years (4-6). Therefore, knowledge of the most common uropathogens and their antibiotic susceptibility patterns in a specific geographic area can be of great help in the selection of the most appropriate agents. The objective of this study was to determine the common uropathogens in admitted patients with UTI and to assess their antibiotic susceptibility patterns.

\section{Patients and Methods}

This retrospective study was conducted on all urinary tract cultures performed during October 2008 to May 2011 at the Department of Pediatrics, Abuzar Children's Hospital, Ahvaz (In south west of Iran). We included all admitted pediatric patients presenting with symptomatic community acquired urinary tract infection.

\subsection{Antimicrobial Susceptibility Testing}

Urine samples with a colony count of $>10^{5}$ (by midstream collection), $>10^{4}$ (by catheter collection), and $>$ $10^{3}$ (by suprapubic collection) were included in the study (7). Cultures with growth of more than 1 organism or fungal infection and samples from patients who received prophylactic antibiotics were excluded from the study. All the samples were cultured for 24 hours at $37^{\circ} \mathrm{C}$ on standard media of blood agar or EMB from Merck and Himedia companies in the autoclave and standard techniques were used for culture and identification of pathogens (8). After bacteria were identified by standard methods, antimicrobial susceptibility testing was performed by the Kirby-Baut method using a panel of antimicrobial agents, including amikacin, gentamicin, ceftriaxone, cefotaxime, cefixime, co-trimoxazole (TMP/SMX), nalidixic acid, and nitrofurantoin. After $24 \mathrm{~h}$, the bacterial isolates were divided into resistant and sensitive groups according to the standard NCCLS table accompanying each disc and we excluded the intermediate reactions (9).

A questionnaire was designed to include data such as age, gender, clinical findings, lab data, and antibiotic susceptibility of bacterial isolates. All the variables were compared using Student's t-test, Mann-Whitney U-test, and chi-square test (for quantitative and qualitative variables, respectively). Quantitative variables are provided as median (with IQR), and $P$ values of $<0.05$ were considered statistically significant.

\section{Results}

A total of 288 urinary specimens of different patients were included in the study. There were 62 boys (21.5\%) and 226 girls (78.5\%) with a median age of 13 months (range, 1 month to 14.5 years; IQR=9-40). About $72 \%$ of males with UTI were aged less than one year. In infants under the age of one year, the number of females with UTI was approximately two times more than that of males (67.1\% versus $32.9 \%$ ). In children above the age of one year, the female: male ratio increased to $8: 1$ ( $89.4 \%$ girls and $10.6 \%$ boys).

Fever was present in $46.9 \%$ of cases, and dysuria, vomiting, and abdominal pain were present in $44.2 \%, 24.8 \%$, and $23.4 \%$, respectively. Abnormal findings on sonography (including hydronephrosis, hydroureter, and bladder hypertrophy) were present in $19 \%$ of patients; vesicoureteral reflux (VUR) and a history of UTI were present in $26.9 \%$ and $19.8 \%$ of patients, respectively ( Table 1 ).

Table 1. Clinical Characteristics in Patients With Urinary Tract Infection

\begin{tabular}{|c|c|c|c|c|}
\hline Findings & $\begin{array}{l}\text { Overall } \\
(\mathbf{n}=\mathbf{2 8 8})\end{array}$ & $\begin{array}{l}\text { Afebrile } \\
(53.2 \%)\end{array}$ & $\begin{array}{l}\text { Febrile } \\
(46.8 \%)\end{array}$ & Pvalue \\
\hline Dysuria & $56.1 \%$ & $57.2 \%$ & $54.9 \%$ & 0.69 \\
\hline Abdominal pain & $23.4 \%$ & $23 \%$ & $25.2 \%$ & 0.68 \\
\hline Vomiting & $24.8 \%$ & $24 \%$ & $28.4 \%$ & 0.4 \\
\hline History of UTI & $19.8 \%$ & $20.9 \%$ & $18.5 \%$ & 0.61 \\
\hline Leukocytosis $^{\mathrm{a}}$ & $37.5 \%$ & $24.5 \%$ & $52.2 \%$ & $<0.001$ \\
\hline Anemia b & $57.9 \%$ & $51 \%$ & $65.7 \%$ & 0.012 \\
\hline Nitrite positive & $66.7 \%$ & $71.6 \%$ & $62.8 \%$ & 0.22 \\
\hline $\begin{array}{l}\text { Decreased spe- } \\
\text { cific gravity }\end{array}$ & $8 \%$ & $7.9 \%$ & $8.1 \%$ & 0.93 \\
\hline VUR & $26.9 \%$ & $26.3 \%$ & $27.5 \%$ & 0.85 \\
\hline Proteinuria $^{\mathrm{d}}$ & $21 \%$ & $14.6 \%$ & $28.1 \%$ & 0.005 \\
\hline Elevated ESR ${ }^{\mathrm{e}}$ & $50.5 \%$ & $27.1 \%$ & $69.2 \%$ & $<0.001$ \\
\hline Positive CRP & $60.3 \%$ & $40 \%$ & $78 \%$ & $<0.001$ \\
\hline
\end{tabular}

The most common pathogens were E. coli (84\%), followed by Klebsiella spp. (10.1\%), Enterococcus spp. (2.4\%), Proteus spp. (1.7\%), and Pseudomonas spp. (1.7\%). Overall bacterial resistance spectrum was highest for TMP/SMX (64\%) and lowest for nitrofurantoin (NF) (10.2\%) (Figure). In 60 patients (21\%), there was no resistance to all our antibiotics, but multidrug-resistant cultures (resistant to more than 3 antibiotics) were seen in 43 cases (15\%). 


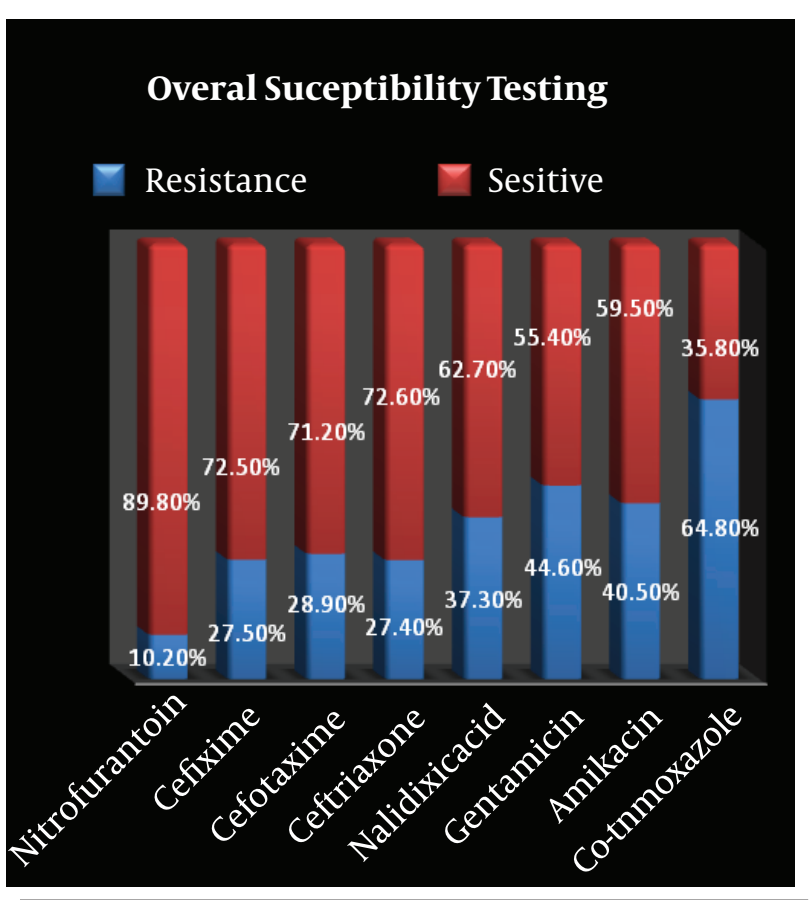

Figure 1. Susceptibility to Nitrofurantoin, Cefixime, Cefotaxime, Cef triaxone, Nalidixic Acid, Gentamicin, Amikacin, Co-trimoxazole, and in 288 Pediatric Urinary Tract Infections Caused by All Uropathogens.
Table 2 shows the resistance patterns of E. coli and Klebsiella spp. against antimicrobial agents. In patients with febrile UTI (46.8\%), E. coli and Klebsiella spp. were the most common pathogens $(86.3 \%$ and $8.1 \%$, respectively). Resistance was the highest to co-trimoxazole and the lowest to nitrofurantoin. Febrile UTI was significantly correlated with anemia $(\mathrm{P}=0.012)$, leukocytosis $(P<0.001)$, proteinuria $(P=0.005)$, increased erythrocyte sedimentation rate $(\mathrm{P}<0.001)$ and $\mathrm{C}$-reactive protein $(\mathrm{P}<0.001)$, but the antibiotic resistance pattern in this group was not significantly different from that in afebrile children.

Among children infected with E. coli and Klebsiella spp. resistance to antibiotics was not correlated with age, gender, and past history of UTI (P>0.05); however, VUR and abnormal sonography were associated with more resistance to cefotaxime $(\mathrm{P}=0.017)$, ceftriaxone $(\mathrm{P}=0.004)$, nitrofurantoin $(\mathrm{P}=0.014)$, and nalidixic acid $(\mathrm{P}<0.001)$. In boys, the mean age was 11.8 mon; $64 \%$ of them were not circumcised, and VUR was found in $37.5 \%$ (vs. $24.2 \%$ in girls). The pattern of resistance to antibiotics in boys was not significantly different from that in girls, but infection with Klebsiella spp. was more common (16.1\% vs. $8.4 \%)$.

Table 2. Resistance patterns of Escherichia coli and Klebsiella spp

\begin{tabular}{llll}
\hline & Overall Resistance, $\%(\mathbf{n}=\mathbf{2 8 8})$ & Escherichia coli $(\mathbf{n}=\mathbf{2 4 2})$ & Klebsiella $\mathbf{s p p} .(\mathbf{n}=\mathbf{2 9})$ \\
\hline Co-trimoxazole & 64.8 & 66 & 56 \\
\hline Amikacin & 40.5 & 39.3 & 42.1 \\
\hline Gentamicin & 44.6 & 44.4 & 40.7 \\
Nalidixicacid & 37.3 & 35.7 & 30.8 \\
Ceftriaxone & 27.4 & 25 & 38.1 \\
Cefotaxime & 28.9 & 25 & 44.4 \\
Cefixime & 27.5 & 22.7 & 71.4 \\
Nitrofurantoin & 10.2 & 4.9 & 24 \\
\hline
\end{tabular}

\section{Discussion}

UTI is always treated empirically before results of bacteriological cultures are available. The choice of antibiotics depends upon the causative organism and its local expected antibiotic susceptibility pattern. We analyzed the spectrum and resistance pattern of uropathogens to common antimicrobial agents and compared them with the results of other recent similar studies.

About $44 \%$ of all strains in our study were resistant to aminoglycosides, making these drugs unsuitable for single therapy in empirical treatment of febrile UTI. There were also high resistance rates to alternative agents such as ceftriaxone (almost 28\%) and oral cefixime (almost $28 \%$ ), which is frequently used for community-acquired UTI in children. The increased resistance rate to amino- glycosides and cephalosporins was presumably due to widespread use of these antibiotics and the unrestricted prescription policy within the last decade.

Resistance rates to TMP/SMX were remarkably high (almost 65\%). This high resistance rate was also observed in many other studies (10-12) and may reflect a possible widespread use of this low-cost antimicrobial agent for treatment and prophylaxis of UTI or as a prophylactic agent against Pneumocystis carinii infections in recipients of immunosuppressive drugs and in the HIV-infected population. As expected, the most common pathogen was E. coli, followed by gram-negative bacteria of the Enterobacteriaceae family, which is in accordance with other studies $(10,13,14)$.

Our study detected much higher resistance rates than those detected by other recent studies (15-19). The Ba- 
hadin et al. study in Singapore recently reported lower resistance rates to ceftriaxone (8.1\%), nalidixic acid (35\%), gentamicin (8.5\%), and TMP/SMX (37.8\%) among E. coli strains from Bedok hospital (15). In another Korean study performed in 2009 to evaluate antimicrobial resistance in UTI, E.coli resistance to TMP/SMX and ceftriaxone was found to be $32.7 \%$ and $5.3 \%$, respectively. (19). The current study found that E. coli resistance to TMP/SMX and ceftriaxone was $64.8 \%$ and $27.4 \%$, respectively; both rates are higher than those reported by the previous studies.

The resistance rate to cephalosporins was significantly higher than other new studies $(15,16,18)$. The worldwide spread of extended spectrum B-lactamases (ESBLs) produced by enteric pathogens is now the most alarming problem and could explain this widespread increase in resistance against $\beta$-lactams. Antibiotic utilization patterns, including widespread cephalosporin use, have been associated with the emergence of ESBLs. More than 200 types of ESBLs have been described in various species of the Enterobacteriaceae family and other non-enteric organisms, including P. aeruginosa and Acinetobacter sp.

ESBLs hydrolyze third-generation cephalosporins and aztreonam but not carbapenems and are inhibited by clavulanic acid and tazobactam. Furthermore, many ESBL-producing pathogens also express plasmid-encoded multidrug resistance (20). Many studies worldwide have reported a noticeable increase in resistance to ciprofloxacin and other fluoroquinolones. Ciprofloxacin is one of the most frequently prescribed fluoroquinolones for UTIs in adults because it has shown excellent activity against pathogens commonly encountered in complicated UTIs (21).

A study from Iran reported that E. coli has a resistance rate of $32 \%$ to ciprofloxacin (18); other recent studies from Singapore and Korea have reported resistance rates of about $25 \%(15,19)$. The resistance rate against nitrofurantoin was significantly low in several recent studies $(15,16)$. It was almost $10.2 \%$ in our study, indicating that nitrofurantoin is a suitable choice for the treatment and prophylaxis of cystitis. However, the high frequency of gastrointestinal side effects limits use of this medicine in children. In our study, urinary tract abnormalities were associated with higher frequency of drug-resistant uropathogen infections; this finding is similar to those of other studies $(13,22)$.

Prophylactic use of antibiotics and a history of antibiotic usage have been mentioned as other risk factors associated with antibiotic resistance $(13,14,23)$. A study from the state of Wisconsin showed a high rate of resistance to third-generation cephalosporins in children receiving prophylactic antibiotics (13); in another study from Turkey, resistance rates for gentamicin and ceftriaxone were higher in patients who received antibiotic prophylaxis (14). This may be due to changes in the intestinal flora and indwelling gram-negative rods with the ability of ESBL production, which is especially important in $K$. pneumoniae and E. coli (12).

In our study, females constituted the majority of children with UTI (77\%), with an 8:1 female to male ratio that is in agreement with previous studies $(15,24)$. The highest frequency of UTI in boys was observed during the first year of life (72\%), but at this age, UTI was 2 times more common in girls; however, this finding may be related to the high frequency of circumcision of boys in our region.

According to our study, increasing resistance to thirdgeneration cephalosporins changed our opinion for using them as single empiric intravenous therapy in hospitalized patients with acute pyelonephritis and in very ill patients; better success will be achieved by concomitant use of an aminoglycoside or using other potent antibiotics. On the other hand, patients with their afebrile community-acquired UTI can be treated more conventionally with oral antibiotics such as nitrofurantoin or an oral cephalosporin, particularly in view of the very low resistance of the most common pathogen E. coli to nitrofurantoin and cefixime in this study. However, restricted use of antibiotics and combination therapy may limit the increasing pattern of antibiotic resistance.

\section{Acknowledgements}

This study was supported by the vice-chancellor of the research center at the Jundishapur University of Medical Sciences (No:U-8832). The authors would like to thank Mr. Cheraghian for his help in the statistical analysis of the results.

\section{Financial Disclosure}

None declared.

\section{Funding/Support}

Ahvaz Jundishapur University of Medical Sciences

\section{Authors' Contribution}

None declared.

\section{References}

1. Haller M, Brandis M, Berner R. Antibiotic resistance of urinary tract pathogens and rationale for empirical intravenous therapy. Pediatr Nephrol. 2004;19(9):982-6.

2. Jakobsson B, Berg U, Svensson L. Renal scarring after acute pyelonephritis. Arch Dis Child. 1994;70(2):111-5.

3. Taneja N, Chatterjee SS, Singh M, Singh S, Sharma M. Pediatric urinary tract infections in a tertiary care center from north India Indian JMed Res. 2010;131:101-5.

4. Bean DC, Krahe D, Wareham DW. Antimicrobial resistance in community and nosocomial Escherichia coli urinary tract isolates, London 2005-2006. Ann Clin Microbiol Antimicrob. 2008;7:13.

5. Brown PD, Freeman A, Foxman B. Prevalence and predictors of trimethoprim-sulfamethoxazole resistance among uropathogenic Escherichia coli isolates in Michigan. Clin Infect Dis. 2002;34(8):1061-6.

6. Shannon KP, French GL. Increasing resistance to antimicrobial agents of Gram-negative organisms isolated at a London teach ing hospital, 1995-2000. J Antimicrob Chemother. 2004;53(5):818- 
25.

7. Ghorashi Z, Ghorashi S, Soltani-Ahari H, Nezami N. Demographic features and antibiotic resistance among children hospitalized for urinary tract infection in northwest Iran. Infect Drug Resist. 2011;4:171-6.

8. Holland TL, Woods CW, Joyce M. Antibacterial susceptibility testing in the clinical laboratory. Infect Dis Clin North Am. 2009;23(4):757-90.

9. Villanova PA. National committee for clinical laboratory standards (NCCLS). Protect lab workers infect. 1990;2.

10. Catal F, Bavbek N, Bayrak O, Karabel M, Karabel D, Odemis E, et al. Antimicrobial resistance patterns of urinary tract pathogens and rationale for empirical therapy in Turkish children for the years 2000-2006. Int Urol Nephrol. 2009;41(4):953-7.

11. Nys S, Terporten PH, Hoogkamp-Korstanje JA, Stobberingh EE. Trends in antimicrobial susceptibility of Escherichia coli isolates from urology services in The Netherlands (1998-2005). J Antimicrob Chemother. 2008;62(1):126-32.

12. Wagenlehner FM, Niemetz A, Dalhoff A, Naber KG. Spectrum and antibiotic resistance of uropathogens from hospitalized patients with urinary tract infections: 1994-2000. Int J Antimicrob Agents. 2002;19(6):557-64.

13. Lutter SA, Currie ML, Mitz LB, Greenbaum LA. Antibiotic resistance patterns in children hospitalized for urinary tract infections. Arch Pediatr Adolesc Med. 2005;159(10):924-8.

14. Yuksel S, Ozturk B, Kavaz A, Ozcakar ZB, Acar B, Guriz H, et al. Antibiotic resistance of urinary tract pathogens and evaluation of empirical treatment in Turkish children with urinary tract infections. Int J Antimicrob Agents. 2006;28(5):413-6.

15. Bahadin J, Teo SS, Mathew S. Aetiology of community-acquired urinary tract infection and antimicrobial susceptibility pat terns of uropathogens isolated. Singapore Med J. 2011;52(6):415-
20.

16. Caracciolo A, Bettinelli A, Bonato C, Isimbaldi C, Tagliabue A, Longoni L, et al. Antimicrobial resistance among Escherichia coli that cause childhood community-acquired urinary tract infections in Northern Italy. Ital J Pediatr. 2011;37:3.

17. Hamdan HZ, Ziad AH, Ali SK, Adam I. Epidemiology of urinary tract infections and antibiotics sensitivity among pregnant women at Khartoum North Hospital. Ann Clin Microbiol Antimicrob. 2011;10:2.

18. Kashef N, Djavid GE, Shahbazi S. Antimicrobial susceptibility patterns of community-acquired uropathogens in Tehran, Iran. J Infect Dev Ctries. 2010;4(4):202-6.

19. Lee SJ, Lee DS, Choe HS, Shim BS, Kim CS, Kim ME, et al. Antimicrobial resistance in community-acquired urinary tract infections: results from the Korean Antimicrobial Resistance Monitoring System. J Infect Chemother. 2011;17(3):440-6.

20. Livermore DM. beta-Lactamases in laboratory and clinical resistance. Clin Microbiol Rev. 1995;8(4):557-84.

21. Astal ZE. Increasing ciprofloxacin resistance among prevalent urinary tract bacterial isolates in the Gaza Strip. Singapore Med J. 2005;46(9):457-60.

22. Allen UD, MacDonald N, Fuite L, Chan F, Stephens D. Risk factors for resistance to "first-line" antimicrobials among urinary tract isolates of Escherichia coli in children. Canadian Med Asso J.1999;160(10):1436-1440.

23. Sotto A, De Boever CM, Fabbro-Peray P, Gouby A, Sirot D, Jourdan J. Risk factors for antibiotic-resistant Escherichia coli isolated from hospitalized patients with urinary tract infections: a prospective study.J Clin Microbiol. 2001;39(2):438-44.

24. Marild S, Jodal U. Incidence rate of first-time symptomatic urinary tract infection in children under 6 years of age. Acto Paediatr. 1998;87(5):549-52. 